\title{
Evaluation of a portable image overlay projector for the visualisation of surgical navigation data: phantom studies
}

\author{
K. Gavaghan · T. Oliveira-Santos - M. Peterhans • \\ M. Reyes · H. Kim · S. Anderegg - S. Weber
}

Received: 29 June 2011 / Accepted: 28 September 2011 / Published online: 21 October 2011

C CARS 2011

\begin{abstract}
Introduction Presenting visual feedback for image-guided surgery on a monitor requires the surgeon to perform timeconsuming comparisons and diversion of sight and attention away from the patient. Deficiencies in previously developed augmented reality systems for image-guided surgery have, however, prevented the general acceptance of any one technique as a viable alternative to monitor displays. This work presents an evaluation of the feasibility and versatility of a novel augmented reality approach for the visualisation of surgical planning and navigation data. The approach, which utilises a portable image overlay device, was evaluated during integration into existing surgical navigation systems and during application within simulated navigated surgery scenarios.

Methods A range of anatomical models, surgical planning data and guidance information taken from liver surgery, cranio-maxillofacial surgery, orthopaedic surgery and biopsy were displayed on patient-specific phantoms, directly on to the patient's skin and on to cadaver tissue. The feasibility of employing the proposed augmented reality visualisation approach in each of the four tested clinical applications was qualitatively assessed for usability, visibility, workspace, line of sight and obtrusiveness.

Results The visualisation approach was found to assist in spatial understanding and reduced the need for sight diversion throughout the simulated surgical procedures.
\end{abstract}

K. Gavaghan · T. Oliveira-Santos $(\bowtie) \cdot$ M. Peterhans $\cdot$ M. Reyes .

H. Kim $\cdot$ S. Anderegg $\cdot$ S. Weber

Institute of Surgical Technology and Biomechanics,

University of Bern, Bern, Switzerland

e-mail: thiago.oliveira@istb.unibe.ch

T. Oliveira-Santos $\cdot$ M. Peterhans $\cdot$ S. Weber

ARTORG Center for Biomedical Engineering Research, CCAS,

University of Bern, Bern, Switzerland
The approach enabled structures to be identified and targeted quickly and intuitively. All validated augmented reality scenes were easily visible and were implemented with minimal overhead. The device showed sufficient workspace for each of the presented applications, and the approach was minimally intrusiveness to the surgical scene.

Conclusion The presented visualisation approach proved to be versatile and applicable to a range of image-guided surgery applications, overcoming many of the deficiencies of previously described AR approaches. The approach presents an initial step towards a widely accepted alternative to monitor displays for the visualisation of surgical navigation data.

Keywords Augmented reality - Image overlay · Projection · Data visualisation · Navigated surgery

\section{Introduction}

The previous two decades have seen an increase in the use of tools for pre-operative planning and intra-operative guidance. Surgical navigation systems and pre-operative planning tools rely on imaging data to assist in the definition and conduct of surgical procedures and in the identification of critical anatomical structures. Patient-specific anatomical models can be constructed from medical image data, allowing surgeons to visualise target and risk structures in 3D alongside surgical plans and guidance information. Pre-operatively, such data aid in the definition of resection planes, trajectories and surgical approaches. Intra-operatively, virtual reality (VR) data registered to the patient displayed alongside guided surgical tools and guidance visual feedback assist in the successful completion of the planned surgical approach, in the localisation of hidden target structures and in the prevention of injury to surrounding tissues. 
Primarily, surgical planning and navigation data are displayed on nearby 2D monitors; however, this approach requires the surgeon to divert his sight and attention between the virtual information on the screen and the patient. Error and excess surgical time introduced due to a lack of intuitiveness and reduced patient attention have called for the development of alternative data visualisation methods. Augmented reality (AR), the fusion of real world and virtual data in a single view, allows such deficiencies to be overcome by displaying navigation data directly in the view of the patient. The advantage of AR to a range of surgical applications has been previously identified and reported.

In navigated soft tissue surgery, the display of 3D models of critical and target structures such as blood vessels and metastases aids in the definition and conduct of surgical procedures. However, authors such as Hansen et al. [1] and Sugimoto et al. [2] have reported on the disadvantages of displaying navigation data on a separate screen. Hansen concluded that mental fusion of planning models with the current surgical view was error-prone and that it frequently resulted in distracting comparisons during the intervention that consumed an unacceptable amount of time. Hansen proposed the idea of using AR technologies such as projection or image overlay for improved visualisation of navigation data but is yet to describe an implemented functional system for doing so. Sugimoto et al. described the use of standard data projectors for $2 \mathrm{D}$ image overlay AR in soft tissue surgery; however, the set-up suffered from a lack of flexibility and a lack of accurate registration techniques and patient movement handling.

In cranio-maxillofacial (CMF) surgery, several authors have described the benefits of AR systems for procedures such as post-traumatic trauma reconstruction [3] and bone resection [4]. Wagner et al. attempted to overlay preoperatively planned resection lines for mandibular osteotomies using head-mounted displays and image overlay technology [4]. The technique provided reasonably accurate image overlay for surgical guidance of resection areas but required complex patient to virtual data registration and intrusive head gear to be worn by all operating physicians.

In [5] and [6], Blackwell and DiGioia et al. described the benefits of applying AR technologies to a range of navigated orthopaedic surgeries including arthroscopy, joint replacement and tumour removal. They proposed the use of semitransparent displays to give the illusion of navigation data floating within the underlying patient. The approach was initially promising but the need for obtrusive equipment around the surgical scene and the associated limited workspace, long set-up times and complex calibrations prevented the widespread use of the approach in navigated orthopaedic or any other form of surgery.

In minimally invasive and percutaneous interventions for which visual feedback is greatly limited, the need for surgical guidance is evident. In [7], Schwald et al. described the application of semi-transparent display AR to minimally invasive surgery; however, the technique suffered from the disadvantages described above in addition to insufficient accuracy. In more recent work, Liao et al. have presented a system for semi-transparent display AR guidance based on autostereoscopic technology [8]. Initial experimental results suggest that such an elaborated system can provide AR to several observers without presenting parallax problems and without additional head-mounted equipment such as displays or trackers. Although such a sophisticated method may present an AR solution for certain minimally invasive procedures, current autostereoscopic displays still suffer from poor image resolution, blind spots along the field of view and cause visual sickness to certain range of observers making it unsuitable for some applications.

In addition, a number of other groups have developed AR approaches based on image overlay [9], semi-transparent displays [5-7], head-mounted displays $[10,11]$ and 2D projection $[2,12,13]$ for specific clinic applications that have been applied with varying success. Overcoming deficiencies in limited workspace, obtrusive equipment requirements, elaborate set-up times and reduced surgical vision appear, however, to be paramount to the widespread acceptance of a single AR approach as an alternative to monitor display in navigated surgery. In addition, a single technique that can be used in a range of image-guided surgeries, without the need to replace existing verified image guidance systems will inevitably enhance the general acceptance of an AR approach.

A portable projection image overlay device (IOD) [14] developed within our institute displays medical data directly on the patient and overcomes many of the deficiencies of previously described AR approaches. The device is minimally intrusive, non-impeding to the view of the surgeon, and efficient in set-up and movement handling. Accurate patient registration and patient and tool tracking are realised through the integration of the device into existing surgical navigation systems with verified registration and tracking frameworks.

In an attempt to evaluate the feasibility and versatility of the image overlay projection device as an alternative tool for surgical planning and navigation data visualisation, we herein present an evaluation of the device within the four surgical applications described above: navigated soft tissue surgery, navigated CMF surgery, navigated orthopaedic tumour resection surgery and the guidance of minimally invasive interventions.

\section{Materials and methods}

Image overlay projection

The image overlay projection device incorporates miniature RGB laser technology (PicoP Microvision, US) that produces 


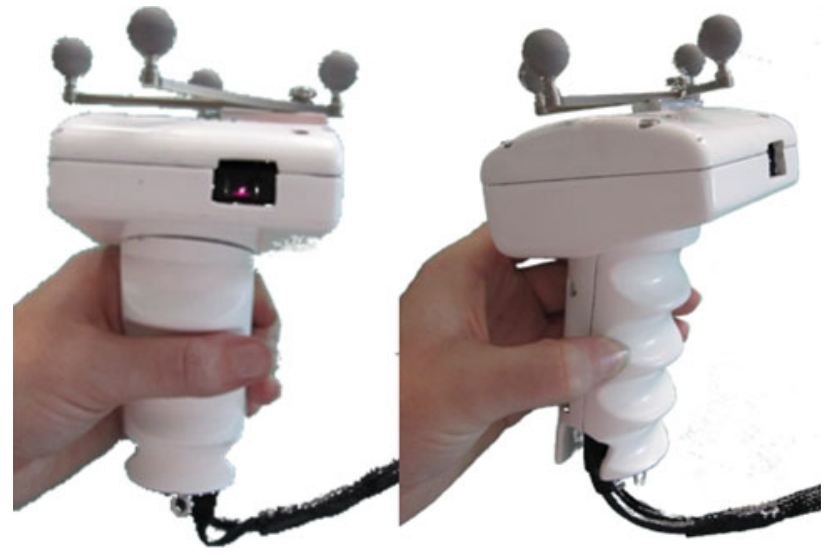

Fig. 1 Image overlay projection device employed for AR visualisation

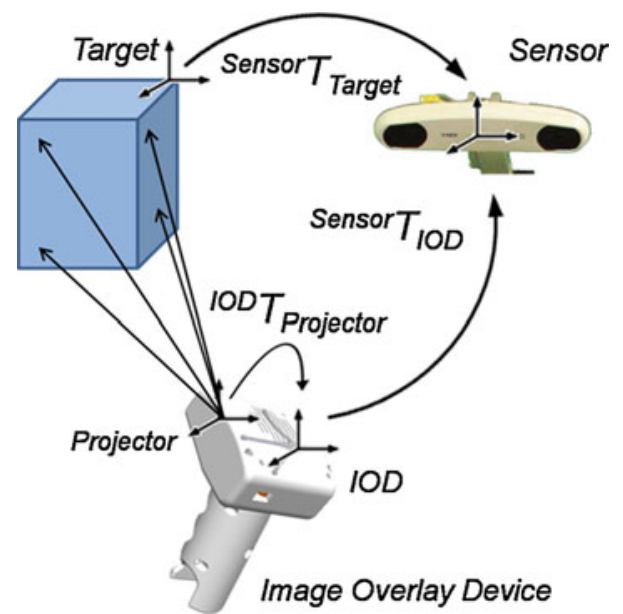

Fig. 2 Image overlay system transformation diagram

in focus images from any projection distance. The device pose is tracked by a navigation system via passive tracking spheres attached to a marker reference on the device housing (Fig. 1). The marker shield is suitable for sterilization in standard hospital reprocessing and can be attached externally to a transparent surgical sterilized drape that covers the entire device and part of the attached cable, making the device suitable for use in sterile clinical environments. The device was designed to be integrated into existing surgical planning and navigation systems in order to utilise the systems' inherent registration and tracking capabilities. The projector has an update rate of $60 \mathrm{~Hz}$ and is thus limited solely by the navigation/planning software image update rate and sensor update rate (typically $20 \mathrm{~Hz}$ ).

By defining a virtual camera in the virtual scene of the surgical planning or navigation software tool, at the pose of the centre of projection, data for projection can be obtained from the view point of the projector (see Fig. 2). Thereafter, geometrically correct images can be projected onto the patient from any position within the navigation camera workspace.
The pose of the centre of projection is calibrated using camera calibration techniques. Projection of the device was described by a reverse pin hole camera model and thus could be solved for the transformation relating an image pixel to a 3D real-world point of projection using Zhang's calibration method [15]. The calibrated transformation defining the static transformation from the projection centre to the tracked marker shield of the device, ${ }^{\mathrm{IOD}} \mathrm{T}_{\text {projector }}$, could thereafter be determined. The pose of the projection centre can be calculated in real time using equation 1 and is used to define the virtual camera perspective within the virtual scene.

${ }^{\text {Sensor }} \mathrm{T}_{\text {Projecor }}={ }^{\text {Sensor }} \mathrm{T}_{\mathrm{IOD}} \cdot{ }^{{ }^{\mathrm{IOD}}} \mathrm{T}_{\text {Projector }}$

Illumination of the device is sufficient to produce images with easily identifiable structures in projected images with size up to $200 \mathrm{~mm} \times 350 \mathrm{~mm}$ in ambient light. As the image overlay device relies on the registration and tracking capabilities of the navigation system into which it is integrated, the accuracy of the device also depends on the accuracy of the navigation system. In a previous study [14], the image overlay device was found to have a mean projection accuracy of $1.3 \mathrm{~mm}$ when integrated into a Liver Surgical Navigation System [16] with navigation camera accuracy of approximately $0.3 \mathrm{~mm}$. Tests were performed on both planar and 3D rigid models. The device is lightweight and portable and has a complete set-up time of less than $2 \mathrm{~min}$. Details of the design, calibration and surface projection accuracy evaluation of the image overlay device are presented in [14]. The image overlay device can be integrated into existing surgical navigation / planning applications via a software module that handles device tracking, image rendering from the perspective of the projector and the output of images for display. To enable AR viewing of existing navigation data, the surgical navigation software must incorporate a 3D virtual scene incorporating anatomical data or guidance data for display. In addition, and as in conventional image-guided surgery, a method of registering the $3 \mathrm{D}$ scene to the real patient must be available.

\section{Surgical guidance system description}

During this study, two surgical guidance systems representative of the systems currently in widespread use were augmented to enable AR viewing. Both systems comprise a navigation computer unit, an infrared-based optical passive tracking system (Vicra, NDI, CA) and touch screen/s for user interaction and visual display. The optical cameras track known configurations of retro-reflective marker spheres. Tools (i.e. ultrasound dissectors, microwave ablation devices, biopsy needles etc) have unique sterilisable reference attachments which the system uses to determine the tools real-time pose. Visual feedback representing the relative pose of the patient anatomy, surgical plans, guidance information and tools is displayed on touch screen monitors. Both systems 
possess an additional DVI interface to which the image overlay device can be attached (Fig. 3). The first system was designed for image guided liver surgery and allows anatomical models, tools and surgical plans to be displayed in a virtual 3D scene. The system incorporates capabilities for both pair point matching and ultrasound based patient-image registration. Patient tracking can be achieved via attachment of a trackable reference. The second system, designed specifically for percutaneous needle intervention guidance based on PET/CT, displays automatically rendered patient models from CT image data in a virtual scene along with coregistered PET images of target lesions. Real-time guidance of a needle to target along a pre-operatively defined trajectory is displayed in the form a cross hair target and depth bar adjacent to the image data. The system incorporates capabilities for both pair point matching and single-marker-based patientimage registration. Patient tracking can be achieved via single-marker tracking or attachment of a trackable reference.

In this study, the first mentioned system was used in the feasibility evaluation of the first three application scenarios in which 3D anatomical models and various surgical planning data were displayed. The later system was used in the feasibility evaluation of minimally invasive intervention AR surface guidance. The systems are described in more detail in [16] and [17], respectively.

\section{Clinical application}

The feasibility and usefulness of the image overlay projector was evaluated in four different clinical scenarios within a laboratory set-up. Integrated into a liver navigation system, the device was used to display anatomical structures such as blood vessels and tumours in addition to resection planes onto patient-specific models and pig liver tissue. In a CMF application, the device was used to display 3D anatomical models for the planning of mandibular tumour resection surgery. In a navigated orthopaedic surgery application, the device was used to project tumour locations and optimal bone resection margins onto patient-specific tibia models. Finally, the device was tested in simulated navigation of a biopsy needle to a target tumour by projecting the target entry point, needle alignment and needle depth directly onto patient skin. Methodology for the generation of image overlay projection in each application is presented below.

\section{Navigated open liver surgery}

The navigation system for open liver surgery described in [16] was augmented to incorporate the image overlay projection device described above. Surface models of a liver and its internal vessels and tumours were pre-segmented from CT by Mevis Distant Services (MeVis Medical Solutions AG, Bremen, Germany) via the process described in [18], see

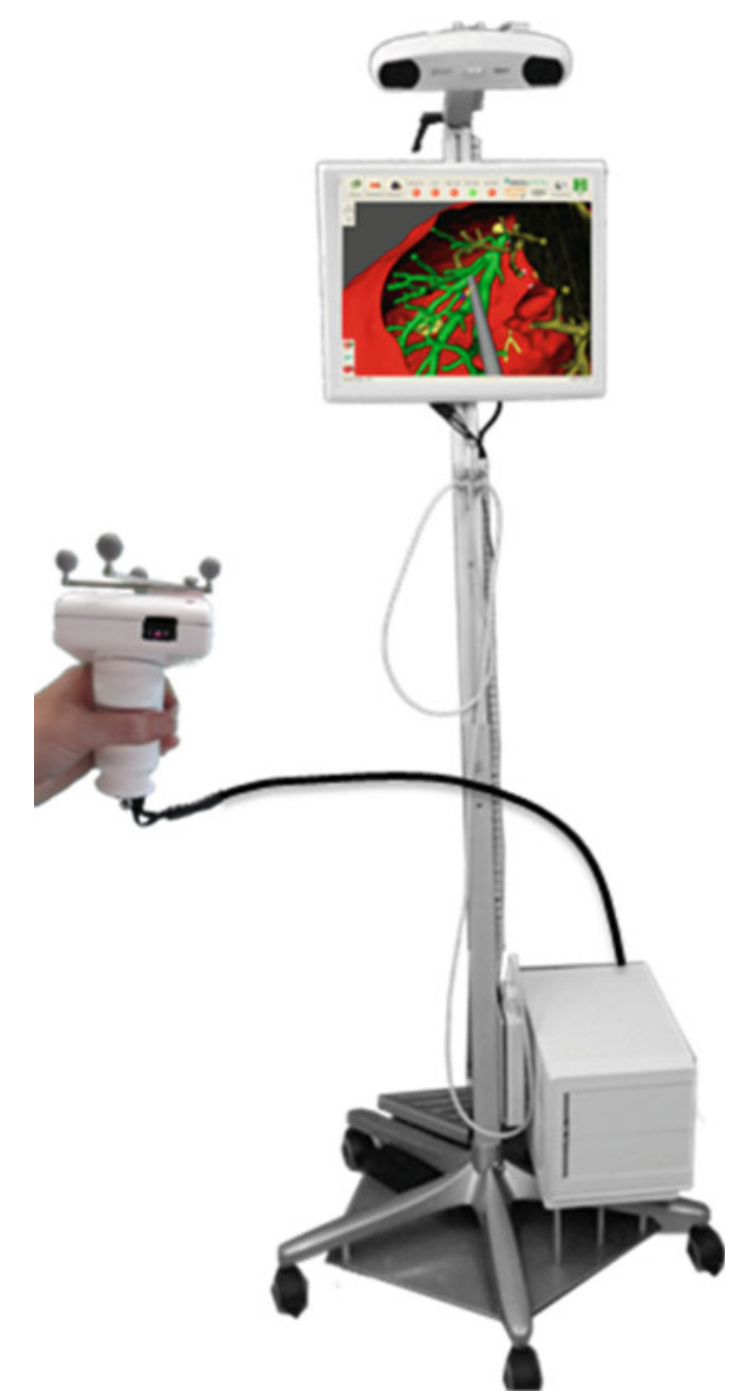

Fig. 3 Stereotactic instrument guidance system with integrated IOD employed within this study

Fig. 4a. The liver model construction process was completed as per non-AR navigated liver surgery, and the models were added to the surgical navigation systems virtual 3D scene as normal. A patient-specific rigid liver model and porcine liver tissue were used as projection surfaces. The rigid model was developed via 3D printing rapid prototyping (Spectrum ZTM 510 printer by ZCorporation). The first was used to validate the projection on the corresponding 3D liver structure, whilst the later was used in the determination of the feasibility of projecting the liver models onto real liver tissue. The liver models were registered to the virtual models with the locally rigid pair point matching technique already implemented in the surgical navigation system.

\section{CMF pre-operative planning and navigated surgery}

The proposed visualisation approach was applied to the planning of a mandibular resection for tumour removal. 3D 


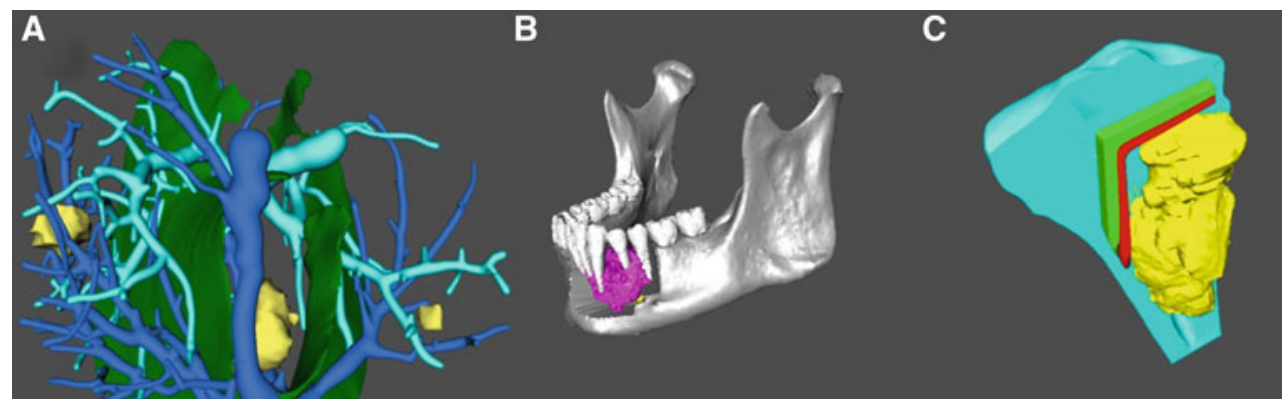

Fig. 4 unmodified original 3D anatomical models from navigation/planning software employed in previous surgical procedures. a Liver resection plan, b CMF osteotomy plan, c proximal tibia resection plan

models of the mandible, target tumour and the mandibular branch of the facial nerve were segmented from NewTom CBCT images using ITK-SNAP as described in [19], see Fig. 4b. For this study, structures were highlighted through increased colour intensity, and the transparency level of the mandible was increased to enable viewing of critical underlying structures in a single view. The right side of the 3D model was removed to prevent overlay interference during projection on the left side of the jaw. Possible resection areas were defined according to tumour position, size and proximity to critical structures. The 3D model with planned resection areas were loaded into the virtual scene of the liver surgical navigation system. A human subject was placed in a sitting position and registered to the virtual data using the navigation systems locally rigid pair point matching capabilities. Conventionally, thereafter, movement of the patient is tracked via a tracking reference, for example, applied to the head and opposite side of the jaw. The 3D anatomical models incorporating various resection options, the tumour tissue and critical structures were projected directly onto the skin of the patient's left jaw.

\section{Navigated orthopaedic tumour resection}

Orthopaedic oncological surgical navigation data including 3D surface models of the proximal tibia, tumour and safe resection margins were projected on a patient-specific rigid 3D phantom developed via 3D printing rapid prototyping. The 3D anatomical models and plan, used previously during image-guided cadaver limb salvage surgery [20], were semi-automatically segmented from CT images using Amir$\mathrm{a}^{\circledR}$ (Visage Imaging, Inc., San Diego, CA,USA) and stored in the form of surface point models and surface meshes, see Fig. 4c. 3D resection planes were added to the anatomical model by the operating surgeon also using Amira ${ }^{\circledR}$. No modification to the original navigation data was made for use in this study. The 3D model with planned safe resection planes was loaded into the virtual scene of the liver surgical navigation system. A 3D rigid phantom of the tibia model was developed via 3D printing rapid prototyping (Spectrum ZTM 510 printer by ZCorporation). The model was registered to its virtual model using the navigation system's locally rigid pair point matching capabilities.

\section{Navigated percutaneous needle intervention}

A navigation system developed to assist percutaneous interventions based on PET/CT images was augmented with the described projection capability, and the feasibility of displaying the necessary guidance information on the patient skin was assessed. For this study, patient CT and PET data were used to simulate a navigated biopsy procedure. A single tumour was isolated in the PET dataset and defined as the target for this procedure. 3D model of the patient surface was automatically generated by the navigation system using contour filters from the Visualization Toolkit (VTK) library. A tracking reference was attached to a band placed around the patient's upper torso to allow for patient movement tracking. Patient to image registration is usually performed automatically by matching the marker spheres from the reference in both spaces, image and tracking. However, in this study, landmark-based registration was used. Prior to the simulated procedure, the user defined the intended needle trajectory by selecting the location of the target and the needle entry point in the navigation system's CT viewers. Guidance information for this application is usually visualised within a targeting viewer that displays the needle tip and shaft positions in addition to a depth bar indicting the distance from the tool tip to the target, see Fig. 5. For this study, the visual information to guide the needle to the target according to the planned trajectory was defined on a plane normal to the patient's skin, centred on the pre-operatively planned entry point. The main cross hair target thus remained static on the patient at the entry sight, whilst the needle pose and depth guidance were updated to the current needle position at a rate of $20 \mathrm{~Hz}$. The correct needle alignment is accomplished by bringing the tip and the shaft to the centre of the cross hair target. The target is reached when the distance bar reaches zero. In the 




Fig. 5 unmodified original biopsy guidance displays from navigation software with patient PET data. a Biopsy needle tip entry site alignment, b biopsy needle shaft alignment, $\mathbf{c}$ biopsy needle depth guidance with visible target lesion (PET data)

Table 1 Data types visualised using AR image overlay projection

\begin{tabular}{llll}
\hline Projected data & & & \\
\hline Application & Anatomy & Plan & $\begin{array}{r}\text { Maximum } \\
\text { projection } \\
\text { size (mm) }\end{array}$ \\
& & & $200 \times 130$ \\
\hline Liver surgery & Vessels, Lesions & Resection planes & $110 \times 80$ \\
CMF surgery & Tumour, Facial nerve, Mandible & Resection area & $90 \times 70$ \\
Orthopaedic Surgery & Tumour & Safe resection margins & $60 \times 80$ \\
Biopsy & Suspicious lesion (PET) & Entry point crosshair & \\
& & Alignment guidance & \\
& & Depth bar & \\
& & Depth in text & \\
\end{tabular}

projected image, PET images were displayed under the cross hair guide to allow visualisation of the target structure as the needle approached. The surgery was emulated in a laboratory set-up using a retractable needle to avoid piercing the skin of the human subject.

\section{Feasibility evaluation}

The feasibility of employing the proposed AR visualisation approach in each clinical application was qualitatively evaluated via the following criteria:

1. Integratability of the device into existing surgical image guidance systems

2. Visibility of the projected structures

3. Obtrusiveness of the device to the surgical scene

4. Workspace of the device due to image size and visibility to the optical tracking camera

5. Usability of the device within the surgical scene
The criteria were assessed during the projection of the anatomical and guidance data summarised in Table 1. The study group for each clinical scenario consisted of three subjects. Two additional observes familiar with the AR approach were present to evaluate the simulated scene. All subjects were familiar with image guidance surgery and optical tracking systems. At least one subject in each group was involved in the development of non-AR image guidance surgical systems for the corresponding clinical application, and at least one subject was unfamiliar with the AR approach being evaluated. Prior to each study, virtual models were loaded into the navigation system, surgical planning was completed and the model or patient was registered to the image data. Thereafter, each simulated projection study followed the below described protocol.

1. The optical tracking system was placed to one side of the patient or phantom model and the three subjects stood in positions of their choice on the opposite side. 
2. One subject was asked to display the image data on the projection surface, whilst remaining subjects identified the structures contained within the projected model.

3. Following structure identification, one subject was instructed to hold the image overlay device in one hand and simultaneously perform simple tasks such as positioning a biopsy needle to its entry alignment or pointing out structures with a scalpel or pointer.

4. Thereafter, one subject was instructed to display the image overlay whilst the two other subjects identified structures with pointers or scalpels or performed a simulated biopsy needle placement using the projected data.

Tasks were repeated until all three subjects with differing skills had operated the image overlay device.

\section{Results}

A software module for handling the tracking of the image overlay device and the generation of images at the pose of the projector was successfully integrated into both navigation systems described above. Minimal modifications were required to existing surgical navigation data to enable their visible projection onto the patient or phantom.

Size of the displayed structures, colour, contrast and illumination were all found to be sufficient to enable individual structures to be identified by all subjects and evaluators in ambient light. The maximum image size of projection for each application, as displayed in Table 1, was within the limit of the device and thus the entire model could be viewed in ambient light in a single projection.

The available workspace was sufficient to allow two additional subjects to perform various tasks without being obstructed by the device and without the subjects obstructing the line of sight between the device and the tracking camera. The device was most commonly held approximately 20 to 30 $\mathrm{cm}$ from the patient approximately perpendicular to the projection surface. Due to its handheld, portable nature and small size, the device could be held in a non-intrusive position and could be easily moved to alternative positions when necessary to remain out of the workspace of the operating subjects. All subjects in all clinical cases were able to hold the device in one hand whilst simultaneously performing simple tasks with the other. Inexperienced subjects were immediately able to use the device in a useful manner. Initially, some operators of the device were more likely to move it quickly throughout the workspace, possibly due to curiosity. Such movements which cause the projected images to be more affected by lag were, however, unnecessary and were no longer performed by operators after a learning period of less than $1 \mathrm{~min}$.

During the feasibility study, the proposed approach allowed underlying anatomical structures and relative resection planes to be viewed in the same view as the patient. $A R$ visualisation of anatomical models along with associated surgical plan data for liver surgery, CMF surgery and orthopaedic surgery performed with the image overlay device are illustrated in Fig. 6. The approach removed the need for the user to mentally align the underlying structures with the real patient or to divert their view or attention away from the patient and thus allowed underlying structures to be identified and targeted intuitively and quickly.

In an open liver surgery, the location of the target tumour tissue could be visualised in relation to surrounding structures directly on the liver surface. Whilst all structures were visible and distinguishable, a certain amount of depth perception was lost in the AR projection and the location of structures was affected by parallax. In CMF surgery, preoperatively defined resection of the mandible could be visualised directly on the patient's jaw. Critical structures in close proximity to the resection area, such as the facial nerve, could be visualised and aided in the planning of a surgical approach. Due to the superficial position of the projected anatomical structures, the visualisation approach suffered minimally from loss of depth perception and parallax effect. In oncology orthopaedics, the image overlay provided an intuitive method of visualising the position of the tumour with respect to the healthy anatomy along with resection safety margins. Locating resection planes on the bone could be achieved intuitively and quickly. Due to the rigidity of bone, the projected images remained immune to distortion that results from tissue deformation. Due to a loss of depth perception, 3D resection planes were, however, more difficult to comprehend suggesting that $2 \mathrm{D}$ resection lines defined on the bone surface itself would be more effectively visualised in such an approach.

During a simulated navigated tumour biopsy procedure, needle position and orientation guidance targets were projected by the image overlay device directly onto the skin of the patient at the pre-operatively defined entry point. Depth information feedback used to guide the needle to the tumour target was displayed adjacent to the guidance target. Text providing precise distance in $\mathrm{mm}$ to the target was displayed below the guidance target. As the simulated position of the needle reached the target, the PET image of the lesion could be viewed in the centre of the cross hair target. Images of the projected guidance are displayed in Fig.7. All information including written text could be easily identified throughout the simulated procedure. Occasionally, projection was obstructed by the biopsy needle itself. Repositioning the projector easily corrected this problem. Displaying such information on the patient skin right at the point of intervention presented certain advantages. It not only facilitated the spatial interpretation required to orient the needle, but also allowed the physician to keep the patient within his field of attention whilst performing the virtual puncture. 

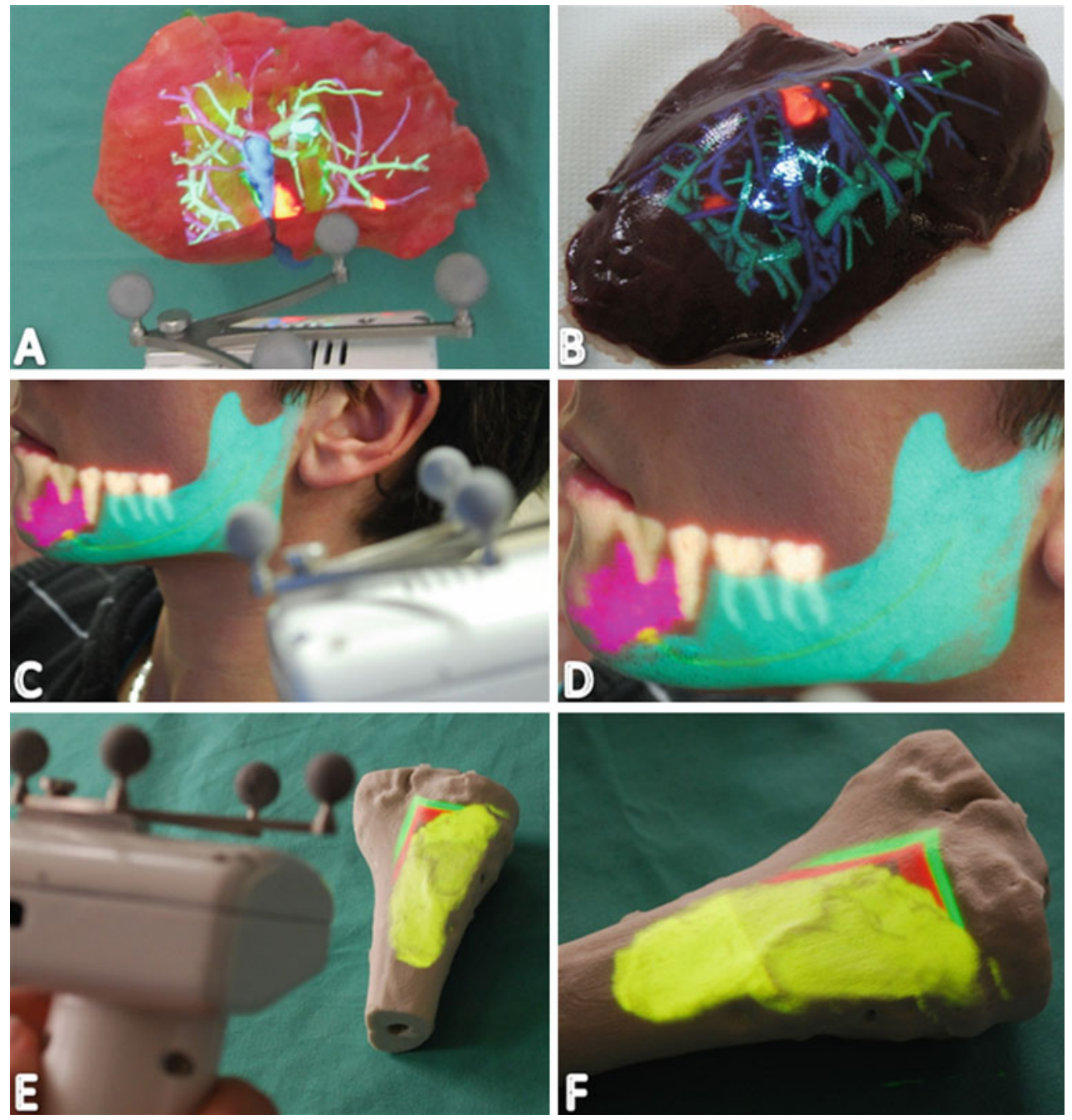

Fig. 6 A Image overlay AR for navigated liver surgery on a patient-specific rigid model and $\mathbf{b}$, pig liver tissue; $\mathbf{c}$, $\mathbf{d}$ image overlay AR for navigated CMF surgical planning; $\mathbf{e}$ and $\mathbf{f}$ image overlay AR for navigated orthopaedic tumour resection

\section{Discussion}

The purpose of this work was to investigate the feasibility of using a novel augmented reality approach for the visualisation of surgical navigation data as an improved alternative to monitor display.

Through the integration of the device and through the projection of a range of existing navigation data, we have demonstrated the versatility of the approach. From our experimental results, we can state that the navigated image projection system can be easily integrated into existing surgical navigation systems and that the proposed visualisation method does not impose any timely or procedural overhead when compared with monitor displayed navigated procedures. The workspace of the approach was sufficient in all four cases to produce easily visible complete scenes on the respective anatomical phantom surface. Additionally, the portable handheld nature of the device allowed the user to maintain line of sight to the optical tracking camera whilst remaining unobtrusive to the surgical scene.

The user benefited from an image augmented view which eliminated the need for diversion of sight or attention during assessment and use of the additional visual information. We believe that such a direct visualisation is highly intuitive, optimises the understanding of the spatial context in which a procedure is carried out and may result in reduced surgical time and increased interventional precision.

Our experiments have demonstrated that in a clinical context, virtually any 3D image can be back-projected to its anatomical origin or the skin above it using this approach. In addition to 3D anatomical models, raw image data such as CT, MRI, angiography or PET images of the critical and target anatomical structures could be displayed. Surgical planning data such as resection planes safety margins, guidance targets, depth levels and even written text can also be displayed directly onto the surgical site. 

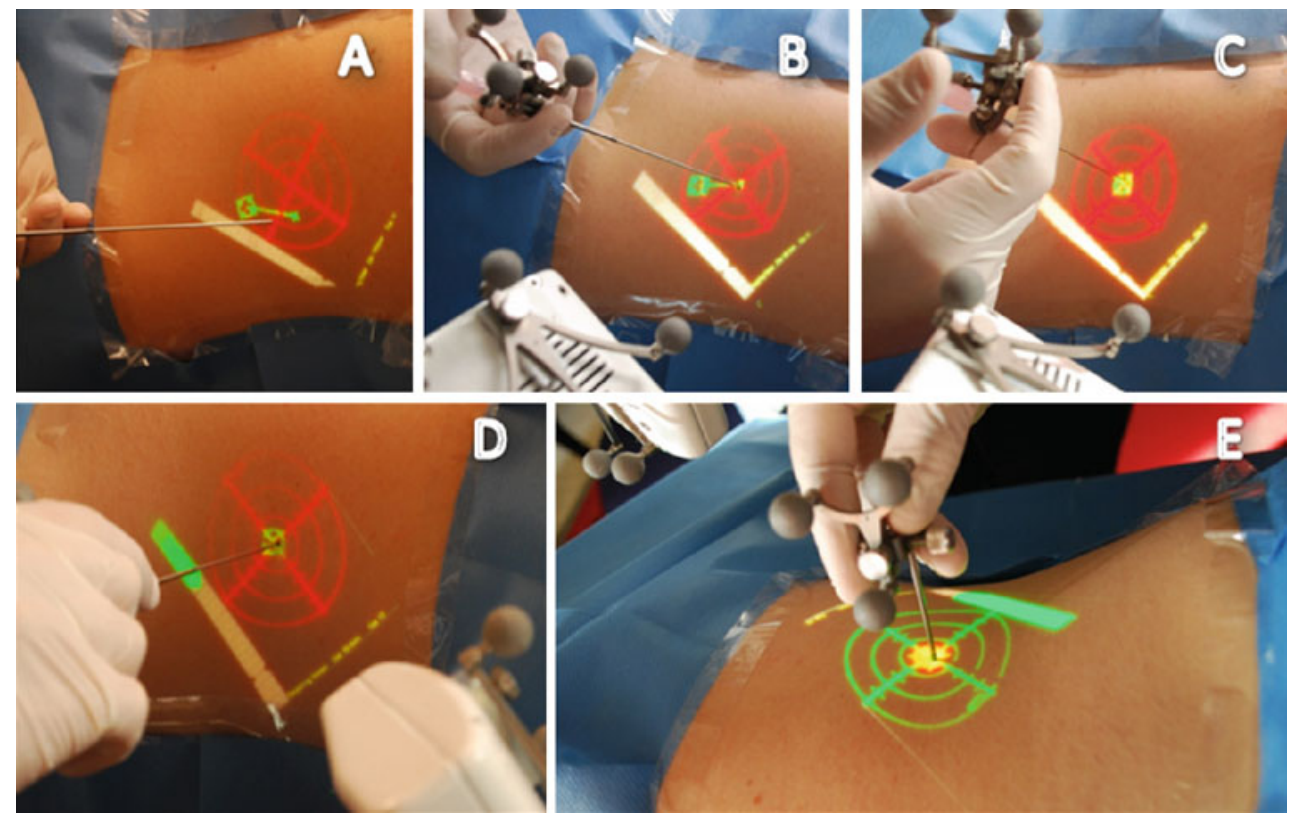

Fig. 7 Image overlay projection of percutaneous needle guidance information throughout a simulated biopsy procedure. a tip alignment, $\mathbf{b}$ shaft alignment, $\mathbf{c}$ aligned needle at point of puncture, $\mathbf{d}$ depth guidance, $\mathbf{e}$ target visualisation

Whilst the benefits of the image overlay for surgical data visualisation were evident, a number of deficiencies in the approach were also identified during this study. Firstly, when projecting onto soft tissue such as skin or the liver surface, deformations in the tissue at the sight of projection can result in distortion of the projected guidance image. In cases of surface guidance such as used in the simulated navigated biopsy, this deficiency can be easily overcome by repositioning guidance data away from the puncture sight after the entry sight has been located and the skin has been penetrated. However, when surgical guidance involves the display of underlying anatomical structures, deformations in the tissue cannot be so easily handled. Handling tissue deformation in this case requires surface tracking of the projection site and appropriate deformation to the projected images in order to maintain correct geometry. Reports from a number of research groups currently exploring soft tissue movement tracking, do however, indicate that higher accuracy solutions are likely to become available in the near future [21-23].

For applications in which all data for visualisation is defined on the patient surface, such as the navigated biopsy, procedure accuracy is not affected by parallax error. All applications displaying structures that are positioned below the projection surface will however be affected by the pose of the viewer. Whilst the parallax error involved in this approach is less than in semi-transparent displays, because the projected scene is viewed directly on the objects surface and not in a certain depth between the observer and the object itself, the effect is still evident when projecting deep lying structures. Previously reported methods of parallax correction have involved non-user-friendly solutions that require head or eye tracking. Requiring the surgeon to wear heavy head gear and limiting their movement to within tracking sensor ranges have been poorly accepted and thus were not integrated into this approach. In future work, we hope to implement a more simplistic and less obtrusive solution to overcome parallax error. Until such time, guiding surgeons to deep targets with surface-defined projections like that displayed in Fig. 7 provide a suitable alternative for procedures with high-accuracy requirements and deeply targeted structures.

Finally, depth perception of displayed data continues to be a challenge in 2D projection. Depth information could be recovered in future applications by coding it into the models being projected as suggested for example by Hansen et al. [1].

In the near future, results of this study suggest that applications involving rigid structures and superficial structures are most suited to AR visualisation using the presented approach. In addition, deep lying structures can be most accurately and quickly target with AR guidance not by displaying the anatomical structure itself but by displaying entrance points, resections lines or real-time guidance applications on the anatomical surface.

In conclusion, we have presented an alternative visualisation method for surgical navigation and planning data that overcomes many of the deficiencies of conventional displays and previously developed AR technologies. We believe that this work highlights the usefulness and feasibility of the approach in a range of surgical applications and thus 
presents an initial step towards a widely accepted alternative to monitor displays for the visualisation of surgical navigation data. It is predicted that future advancements in navigation technology, 3D data representation and miniature laser technology will see continued developments in the presented approach.

Acknowledgments The authors would like to acknowledge Dr. Lucas Ritacco for his contribution to the development of the orthopaedic models used within this study.

Conflict of interest None.

\section{References}

1. Hansen C, Wieferich J, Ritter F, Rieder C, Peitgen H-O (2010) Illustrative visualization of $3 \mathrm{D}$ planning models for augmented reality in liver surgery. Int $\mathrm{J}$ Comput Assist Radiol Surg 5: 133-141

2. Sugimoto M, Yasuda H, Koda K, Suzuki M, Yamazaki M, Tezuka T, Kosugi C, Higuchi R, Watayo Y, Yagawa Y, Uemura S, Tsuchiya H, Azuma T (2010) Image overlay navigation by markerless surface registration in gastrointestinal, hepatobiliary and pancreatic surgery. J Hepato Biliary Pancreat Sci 17:629-636

3. Weber S, Klein M, Hein A, Krueger T, Lueth T, Bier J (2003) The navigated image viewer-evaluation in maxillofacial surgery. Medical image computing and computer-assisted interventionMICCAI 2003, lecture notes in computer science, vol 2878, pp 762-769

4. Wagner A, Rasse M, Millesi W, Ewers R (1997) Virtual reality for orthognathic surgery: the augmented reality environment concept. J Oral Maxillofac Surg 55: 456-462 (discussion 462-463)

5. Nikou C, Digioia A, Blackwell M, Jaramaz B, Kanade T (2000) Augmented reality imaging technology for orthopaedic surgery. Oper Techniq Orthopaed 10:82-86

6. Blackwell M, Morgan F, DiGioia AM (1998) Augmented reality and its future in orthopaedics. Clin Orthopaed Related Res 354:111-122

7. Schwald B, Seibert H, Schnaider M, Wesarg S, Roddiger S, Dogan S (2004) Implementation and Evaluation of an Augmented Reality System Supporting Minimal Invasive Interventions. Workshop AMI-ARCS. Online Proceedings : Augmented Environments for Medical Imaging, 2004, pp. 41-48

8. Liao H, Ishihara H, Tran HH, Masamune K, Sakuma I, Dohi T (2010) Precision-guided surgical navigation system using laser guidance and 3D autostereoscopic image overlay. Comput Med Imaging Graph 34:46-54

9. Marescaux J, Rubino F, Arenas M, Mutter D, Soler L (2004) Augmented-reality-assisted laparoscopic adrenalectomy. JAMA 292: 2214-2215
10. Fuchs H, State A, Yang H, Peck T, Lee SW, Rosenthal M, Bulysheva A, Burke C (2008) Optimizing a head-tracked stereo display system to guide hepatic tumor ablation. Stud Health Technol Inform 132:126-131

11. Sauer F, Khamene A, Bascle B, Schinunang L, Wenzel F, Vogt S (2001) Augmented reality visualization of ultrasound images: system description, calibration, and features. In: Proceedings IEEE and ACM international symposium on augmented reality. IEEE Computer Society, pp 30-39

12. Volonte F, Bucher P, Pugin F, Carecchio A, Sugimoto M, Ratib O, Morel P (2010) Mixed reality for laparoscopic distal pancreatic resection. Int J Comput Assist Radiol Surg 5:126-127

13. Tardif J-P, Roy S, Meunier J (2003) Projector-based augmented reality in surgery without calibration. In: Proceedings of the 25th annual international conference of the IEEE engineering in medicine and biology society, vol 1, pp 548-551

14. Gavaghan KA, Peterhans M, Oliveira-Santos T, Weber S (2011) A portable image overlay projection device for computer-aided open liver surgery. in: IEEE Trans Biomed Eng 58:1855-1864

15. Zhang Z (2000) A flexible new technique for camera calibration. in: IEEE Trans Pattern Anal Mach Intell 22:1330-1334

16. Peterhans $M$, vom Berg A, Dagon B, Inderbitzin D, Baur C, Candinas D, Weber S (2011) A navigation system for open liver surgery: design, workflow and first clinical applications. Int J Med Robotics Comput Assist Surg 7:7-16

17. Oliveira-Santos T, Klaeser B, Weitzel T, Krause T, Nolte L-peter, Peterhans M, Weber S (2011) A navigation system for percutaneous needle interventions based on PET/CT images: Design, workflow and error analysis of soft tissue and bone punctures. Comput Aided Surg 16:203-219

18. Schenk A, Zidowitz S, Bourquain H, Hindennach M, Hansen C, Hahn HK, Peitgen H-O (2008) Clinical relevance of model based computer-assisted diagnosis and therapy. In: Proceedings of SPIE, vol 6915, p 691502. doi:10.1117/12.780270

19. Tucker S, Cevidanes LHS, Styner M, Kim H, Reyes M, Proffit W, Turvey T (2010) Comparison of actual surgical outcomes and 3-dimensional surgical simulations. J Oral Maxill Surg 68: 2412-2421

20. Bou Sleiman H, Ritacco LE, Aponte-Tinao L, Muscolo DL, Nolte L-P, Reyes M (2011) Allograft selection for transepiphyseal tumor resection around the knee using three-dimensional surface registration. Ann Biomed Eng 39:1720-1727

21. Markert M, Koschany A, Lueth T (2010) Tracking of the liver for navigation in open surgery. Int J CARS 5:229-235

22. Oliveira-Santos T, Peterhans M, Hofmann S, Weber S (2011) Passive single marker tracking for organ motion and deformation detection in open liver surgery. In: Taylor RH, Yang G-Z (eds) Information processing in computer-assisted interventions, Berlin, Germany. Springer, Berlin pp 156-167

23. Cash DM, Miga MI, Sinha TK, Galloway RL, Chapman WC (2005) Compensating for intraoperative soft-tissue deformations using incomplete surface data and finite elements. in: IEEE Trans Med Imaging 24:1479-1491 